\title{
Vigilancia de insectos de importancia en salud pública durante la construcción de los proyectos hidroeléctricos Porce II y Porce III, Antioquia, Colombia, 1990-2009
}

\author{
Walter Alonso Zuluaga', Yolanda Lucía López¹, Lisardo Osorio', Luis Fernando Salazar¹, \\ Marta Claudia González ${ }^{1}$, Claudia María Ríos ${ }^{2}$, Marta Isabel Wolff ${ }^{3}$, José Pablo Escobar ${ }^{1}$ \\ 1 Facultad Nacional de Salud Pública, Universidad de Antioquia, Medellín, Colombia \\ 2 Fiocruz AM, Biodiversidade em Saúde, Instituto Leônidas e Maria Deane, Manaus, Amazonas, Brasil \\ ${ }^{3}$ Facultad de Ciencias Exactas y Naturales, Instituto de Biología, Universidad de Antioquia, Medellín, Colombia
}

Introducción. Los estudios entomológicos en las grandes obras de infraestructura hidroeléctrica constituyen una herramienta para la prevención y el control de enfermedades transmitidas por vectores, debido a que con frecuencia las alteraciones causadas en el medio producen aumento de criaderos naturales y artificiales en el área de influencia y, por ende, incremento de las poblaciones de artrópodos, entre ellos, insectos de interés en salud pública.

Objetivo. Realizar estudio y vigilancia de la fauna de Culicidae y Phlebotominae en el área de los proyectos hidroeléctricos Porce II y Porce III, 1990-2009.

Materiales y metódos. Se realizaron muestreos entomológicos periódicos para la vigilancia en salud pública de las comunidades ubicadas en el área de influencia y en campamentos y frentes de obra. Los adultos fueron capturados con red para mariposas, trampas de luz Shannon y CDC, y cebo humano protegido.

Resultados. Se encontraron larvas de mosquitos de Culex coronator, Cx. nigripalpus, Cx. corniger, Cx. quinquefasciatus y Limatus durhami. Los depósitos más frecuentes fueron: tanques bajos, canecas, llantas y matas sembradas en agua. Aedes aegypti solo fue capturado en dos localidades rurales de dos municipios del área de influencia. En las zonas de bosque se capturaron mosquitos Aedes, Mansonia, Culex, Psorophora, Wyeomyia, Phonyomyia, Uranotaenia, Haemagogus y Sabethes; el principal fue Haemogogus janthinomis, eficiente vector de fiebre amarilla en Colombia. La zona es endémica para leishmaniasis y se identificaron 20 especies de Lutzomyia. Entre los vectores de malaria, las principales especies encontradas fueron Anopheles nuñeztovari y An. pseudopunctipennis.

Conclusión. En la zona de Porce II y Porce III existe diversidad de vectores de importancia en salud pública, que es necesario continuar vigilando para minimizar el riesgo de transmisión de enfermedades a los trabajadores de las obras y comunidades aledañas.

Palabras clave: vectores de enfermedades, Anopheles, Psychodidae, Culicidae, presas, centrales eléctricas, salud pública, impacto ambiental.

doi: http://dx.doi.org/10.7705/biomedica.v32i3.668

Twenty-year surveillance of insects relevant to public health during the construction of hydroelectric facilities in Antioquia, Colombia

Introduction. Entomological studies conducted in large hydroelectric infrastructure projects are a tool for the prevention and control of vector-borne diseases. These diseases emerge as a consequence of changes made to the terrain that often increase the natural and artificial mosquito larval habitats. Many

\footnotetext{
Contribución de los autores:

Walter Zuluaga: investigador principal, muestreo e identificación de especies de mosquitos y Lutzomyia spp. entre 2008-2009; recolección y análisis de datos.

Yolanda López: coordinadora del proyecto en la década del 2000, muestreos de campo, identificación de especies en ambas décadas; recolección y análisis de datos.

Lisardo Osorio: muestreo e identificación de especies de mosquitos, década del 90; aporte en discusión.

Luis Fernando Salazar: muestreo e identificación de especies de mosquitos y Lutzomyia spp. entre 2005-2007; aporte en consolidación de datos y referencias.

Claudia González: muestreos de campo en la década del 2000; aporte en resultados.

Claudia Ríos: muestreo e identificación de especies 1999-2000; aporte en resultados.

Marta Wolff: identificación de especies de Lutzomyia década del 90; aporte en resultados.

José Pablo Escobar: coordinador del proyecto y orientación de trabajo de campo, década del 90; análisis de información.
} 
of these insects are of public health importance and population increases result in an increased risk of disease transmission.

Objective. The culicine (mosquito) and phlebotomine (sand fly) populations were characterized in the area of the Porce II and Porce III hydroelectric projects of Antioquia between 1990 to 2009.

Materials and methods. Periodical entomological samplings were made in the area of impact, in the workers camps, and construction sites. Adult specimens were captured with nets, Shannon light traps, CDC light traps, and protected human bait.

Results. Mosquito larvae of the following species were identified: Culex coronator, Culex nigripalpus, Culex corniger, Culex quinquefasciatus and Limatus durhami. The most frequently identifiers of larval habitats were low tanks, waste cans, tires, and aquatic plants. Aedes aegypti specimens were captured in only two rural locations from two municipalities within the area of influence. Specimens from the following mosquito genera were captured in forest areas: Aedes, Mansonia, Culex, Psorophora, Wyeomyia, Phonyomyia, Uranotaenia, Haemagogus and Sabethes. The most important mosquito found was Haemogogus janthinomis, an efficient yellow fever vector in Colombia. The area has been endemic for leishmaniasis and in the current study, 20 species of Lutzomyia sand flies, potential vectors, were identified. Among malaria vectors, the most important species found in the area were Anopheles nuneztovari and Anopheles pseudopunctipennis.

Conclusion. A wide variety of vectors were discovered in the area of the Porce II and Porce III hydroelectric projects, and many of these were relevant for public health. Further monitoring will be necessary to minimize disease transmission risks among the workers and the inhabitants of the surrounding areas.

Key words: Disease vectors, Anopheles, Psychodidae, Culicidae, dams, power plants, public health, environmental impact.

doi: http://dx.doi.org/10.7705/biomedica.v32i3.668

La construcción de plantas hidroeléctricas y embalses genera efectos sobre la salud y el bienestar de las poblaciones de las áreas de influencia, debido a la modificación de las condiciones ambientales y a fenómenos sociales y económicos que pueden causar cambios en las condiciones de vida y aumentar el riesgo del incremento en la propagación de algunas enfermedades. Uno de los efectos es la proliferación de insectos de importancia en salud pública y de enfermedades transmitidas por éstos, entre ellos, los géneros Anopheles (vectores de malaria), Psorophora y Mansonia (vectores de arbovirus) (1).

A su vez, los fenómenos de deforestación en las áreas aledañas a la construcción de plantas hidroeléctricas, también generan fragmentación de hábitats y desplazamiento de mamíferos silvestres, que constituyen un riesgo para los trabajadores y poblaciones vecinas, ante el contacto con transmisores de enfermedades como las moscas del género Lutzomyia (transmisor de leishmaniasis), entre otros artrópodos.

\footnotetext{
Correspondencia:

Yolanda Lucía López, Facultad Nacional de Salud Pública, Universidad de Antioquia, Calle 62 № 52-59, Medellín, Colombia

Teléfonos: (094) 2196819 y 219 6806; fax (094) 2196850

yola@guajiros.udea.edu.co
}

Recibido: 22/07/11; aceptado:12/03/12
Durante la construcción del proyecto hidroeléctrico Porce II, en Antioquia, se registró principalmente la aparición de brotes de malaria, de intoxicación por alimentos y de hepatitis $A$, tanto en la población trabajadora como en personas de las comunidades aledañas (2).

Posteriormente, se han llevado a cabo otros estudios que han dado continuidad a este estudio para la vigilancia epidemiológica en las zonas de construcción de proyectos hidroeléctricos en el departamento. Así, este estudio presenta los resultados del trabajo de la Facultad Nacional de Salud Pública de la Universidad de Antioquia, mediante contrato con las Empresas Públicas de Medellín, referente al registro de la fauna de insectos de importancia en salud pública y a la vigilancia entomológica de vectores de enfermedades en 1990 (antes de la construcción de Porce II) y durante la construcción de los proyectos hidroeléctricos Porce II (1997-2000) y Porce III (2005-2009).

\section{Localización del área}

La central hidroeléctrica Porce II se construyó entre 1994 y 2000 y Porce III se construyó entre 2004 y 2011. Ambas están distantes 140 km de Medellín, capital del departamento de Antioquia, por la carretera que conduce a los municipios de Amalfi y Anorí, en el nordeste del departamento. 
La principal fuente de productividad económica de las poblaciones es el río Porce, donde se explota minería de aluvión, y en menor escala, ganadería y agricultura. El río Porce es la fuente para los dos proyectos en serie, dentro del plan de expansión del sistema energético de las Empresas Públicas de Medellín.

En el área existen dos zonas de vida: 1) bosque húmedo tropical, donde se localiza la mayor parte del área del embalse Porce II, y, 2) bosque muy húmedo premontano, que cubre la parte superior del área de este embalse y de Porce III. La temperatura media es de $22,1^{\circ} \mathrm{C}$, con temperatura máxima de $33,4{ }^{\circ} \mathrm{C}$ y mínima de $13,9{ }^{\circ} \mathrm{C}$. La humedad relativa promedio anual es $83,3 \%$ y la precipitación anual oscila entre 2.300 y $3.300 \mathrm{~mm}$. La zona tiene alturas entre 369 y 1.550 msnm.

El área de influencia de Porce II se estableció siguiendo el curso del río Porce, entre los municipios de Gómez Plata y Guadalupe, y lateralmente, a una distancia de dos kilómetros en la margen izquierda del municipio de Gómez Plata y de cuatro kilómetros en la margen derecha de los municipios de Yolombó y Amalfi (3). En la actualidad, es un embalse con capacidad de 149,37 millones de $\mathrm{m}^{3}$ que inunda 890 hectáreas. La construcción de Porce III se llevó a cabo entre 2004 y 2011 , y su área de influencia se encuentra en algunas veredas de los municipios de Anorí y Amalfi, con una capacidad instalada de 660 megavatios (figura 1).

\section{Materiales y métodos}

Los muestreos se llevaron a cabo en cuatro etapas, tres de ellas con duración de varios años, con diferentes métodos de captura y dirigidos a hacer el inventario de la fauna de especies de la zona y la vigilancia entomológica de los vectores de enfermedades (cuadro 1).

Los sitios de toma de muestras se determinaron teniendo en cuenta: las poblaciones humanas del área de influencia, los pisos de altitud, la cobertura vegetal, la cercanía a los frentes de obra y la percepción de los trabajadores sobre sitios donde picaban los insectos.

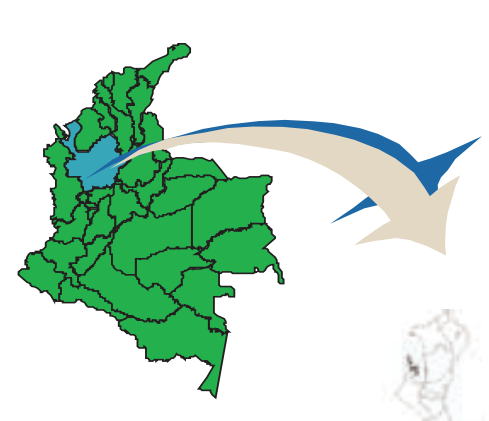

Colombia
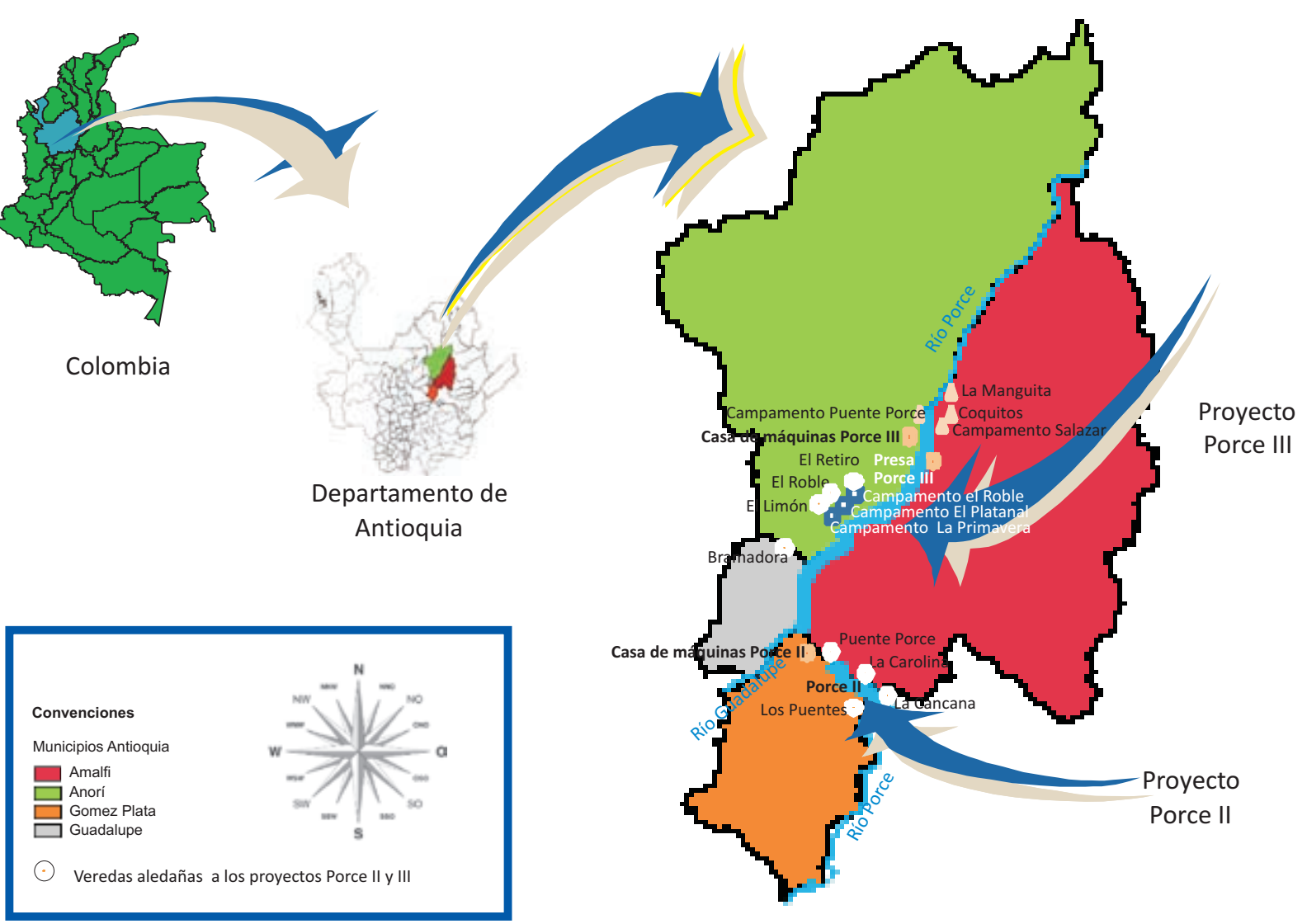

Figura 1. Ubicación geográfica de los proyectos hidroeléctricos Porce II y Porce III y de algunos sitios de muestreo 
Sus coordenadas son las siguientes:

\begin{tabular}{|c|c|c|}
\hline Proyecto & Sitios de muestreo & Coordenadas \\
\hline \multirow[t]{4}{*}{ Porce II } & Vereda Puente Porce (sector Presa Porce 2) & N 06 ${ }^{\circ} 48.618^{\prime} \mathrm{W} 075^{\circ} 08.531^{\prime}$ \\
\hline & Vereda Los Puentes & N 0646.608' W $075^{\circ} 05.299^{\prime}$ \\
\hline & Vereda La Carolina & N $06^{\circ} 47.141^{\prime} \mathrm{W} 075^{\circ} 05.427^{\prime}$ \\
\hline & Corregimiento La Cancana & N 06² $46.481^{\prime} W 075^{\circ} 05.070^{\prime}$ \\
\hline \multirow[t]{15}{*}{ Porce III } & Vereda La Bramadora & N 0652.054' W 075¹0.699' \\
\hline & Vereda El Limón & N 0655.691' W $075^{\circ} 08.650^{\prime}$ \\
\hline & Vereda El Roble & N 06⒌ $58.329^{\prime}$ W $075^{\circ} 07.841^{\prime}$ \\
\hline & Vereda El Retiro & N 0658.213' W $075^{\circ} 07.193^{\prime}$ \\
\hline & Campamento La Primavera & N 0658.418' W $075^{\circ} 05.677^{\prime}$ \\
\hline & Campamento Tocci & 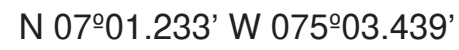 \\
\hline & Campamento Salazar & N 0658.117' W $075^{\circ} 23.036^{\prime}$ \\
\hline & Campamento Alcides Martínez & N 0658.545' W 0750ㅜ.134' \\
\hline & Campamento El Platanal & N 0658.503' W 0750․ $06.781^{\prime}$ \\
\hline & Campamento El Castillo & N 0657.358' W 0750․ $06.411^{\prime}$ \\
\hline & Frente obra La Presa & N $06^{\circ} 55.875^{\prime} \mathrm{W} 075^{\circ} 08.326^{\prime}$ \\
\hline & Casa máquinas & N 0701.190' W $075^{\circ} 03.654^{\prime}$ \\
\hline & Frente obra ventana 3 & N 0700.871' W $075^{\circ} 04.139^{\prime}$ \\
\hline & Frente obra Coquitos & N $07^{\circ} 02.523^{\prime} \mathrm{W} 075^{\circ} 02.497^{\prime}$ \\
\hline & $\begin{array}{l}\text { Bosque Puente Porce (cerca de casa de máquinas } \\
\text { de Porce 3) }\end{array}$ & N 0701.382' W $075^{\circ} 03.498^{\prime}$ \\
\hline
\end{tabular}

La captura de mosquitos diurnos se hizo en los bosques secundarios aledaños a los campamentos y comunidades de las veredas vecinas de Porce II y Porce III, con red para mariposas entre las 10:00 y 12:00, y las 15:00 y 17:30, por dos personas que usaban manga larga y pantalón largo.

La captura de Lutzomyia spp. se hizo en 1990 para el levantamiento de las especies en los sitios especificados en el cuadro $2 \mathrm{y}$, en Porce III, se llevaron a cabo en el período 2004-2009, en 15 puntos de muestreo ubicados entre los 300 y 1.441 msnm, en los sitios especificados en el cuadro 3. La recolección de ejemplares se llevó a cabo durante una o dos noches en cada lugar, con trampa de luz CDC entre las 18:00 y 06:00, con trampa de luz Shannon entre las 18:00 y 21:00 y con cebo humano protegido entre las 18:00 y 21:00. Para la captura en cebo humano, dos personas investigadoras del estudio con protección adecuada, camisa de manga larga y pantalón largo y con repelente en las partes expuestas (manos, cara y cuello), se sentaron a esperar la llegada de los insectos, los cuales fueron recolectados con aspirador bucal y ayuda de una linterna (4). En ningún momento se emplearon auxiliares de campo ni personas de la comunidad para la recolección de insectos.

Los especímenes se identificaron en el Laboratorio de Salud Pública, mediante claves dicotómicas de González y Carrejo, 2009, Lane, 1953, Tinker, 1980, Young, 1982, y Forattini, 2002 (5-9), y la denominación de géneros se hizo de acuerdo con el Systematic Catalog of Culicidae (10).

\section{Muestreo de Aedes aegypti y otros mosquitos}

En Porce II, en 1990, se hizo el levantamiento de índices de infestación larvarios por Ae. aegyptien las localidades del área de influencia (La Carolina, Los Puentes, La Cancana, El Bosque, Puente Porce). Entre 1996 y 2000, se ejerció vigilancia en los campamentos de trabajadores y en los principales frentes de obra (presa y casa de máquinas).

En Porce III, se hizo levantamiento de índices en los caseríos ubicados sobre la carretera que conduce de Medellín a Anorí (veredas Bramadora, El Limón, El Roble y El Retiro), y en campamentos $y$ frentes de obra.

La búsqueda de larvas se llevó a cabo con el método del cucharón en llantas, tarros, canecas, tanques y depresiones del terreno con agua estancada, mediante succión con pipeta en huecos de piso y árboles, y mediante vaciado en cucharon para inspección de botellas. El muestreo se hizo en una de cada dos viviendas de las comunidades, y en los campamentos y frentes de obra. Las larvas se introdujeron en tubo plástico con etanol al $70 \%$, rotulado con datos del sitio, tipo de criadero, fecha 
Cuadro 1. Muestreos entomológicos según etapas del proyecto hidroeléctrico, Antioquia, Colombia, 1990-2009

\begin{tabular}{|c|c|c|c|c|c|}
\hline Proyecto & $\begin{array}{c}\text { Etapa y } \\
\text { años de } \\
\text { muestreo }\end{array}$ & Lugares & $\begin{array}{l}\text { Periodicidad del } \\
\text { muestreo }\end{array}$ & Muestreo para: & $\begin{array}{l}\text { Método de } \\
\text { muestreo }\end{array}$ \\
\hline Porce II & $\begin{array}{l}1990 \\
\text { (antes de inversión } \\
\text { al proyecto) }\end{array}$ & $\begin{array}{l}\text { Comunidades de } \\
\text { influencia }\end{array}$ & $\begin{array}{l}\text { Muestreos mensuales } \\
\text { para levantamiento de } \\
\text { especies, durante } \\
\text { seis meses }\end{array}$ & $\begin{array}{l}\text { Aedes aegypti } \\
\text { Mosquitos silvestres } \\
\text { diurnos } \\
\text { Lutzomyia } \\
\text { Anopheles }\end{array}$ & $\begin{array}{l}\text { Levantamiento de } \\
\text { índices } \\
\text { Dos redes de } \\
\text { mariposas, dos } \\
\text { personas } \\
\text { Dos trampas CDC } \\
\text { Muestreo larvario } \\
\text { con cucharón, dos } \\
\text { trampas CDC }\end{array}$ \\
\hline Porce II & $\begin{array}{l}1996-2000 \\
\text { (construcción de } \\
\text { obras principales) }\end{array}$ & $\begin{array}{l}\text { Campamentos y } \\
\text { frentes de obra }\end{array}$ & Trimestral & $\begin{array}{l}\text { Aedes aegypti } \\
\text { Mosquitos de } \\
\text { recipientes domésticos }\end{array}$ & $\begin{array}{l}\text { Levantamiento de } \\
\text { índices } \\
\text { Muestreo larvario } \\
\text { con cucharón }\end{array}$ \\
\hline Porce III & $\begin{array}{l}2004-2006 \\
\text { (construcción de } \\
\text { obras civiles y de } \\
\text { infraestructura) }\end{array}$ & $\begin{array}{l}\text { Comunidades de } \\
\text { influencia, } \\
\text { campamentos y } \\
\text { frentes de obra }\end{array}$ & Trimestral & $\begin{array}{l}\text { Levantamiento de } \\
\text { Mosquitos de } \\
\text { regimientos } \\
\text { domésticos } \\
\text { Mosquitos silvestres } \\
\text { diurnos } \\
\text { Lutzomyia } \\
\text { Anopheles }\end{array}$ & $\begin{array}{l}\text { Levantamiento de } \\
\text { índices } \\
\text { Muestreo larvario } \\
\text { con cucharón } \\
\text { Dos redes de } \\
\text { mariposas, dos } \\
\text { personas } \\
\text { Cuatro trampas } \\
\text { CDC* } \\
\text { Cebo humano } \\
\text { protegido dos } \\
\text { personas } \\
\text { Muestreo larvario } \\
\text { con cucharón } \\
\text { cuatro trampas } \\
\text { CDC* }\end{array}$ \\
\hline Porce III & $\begin{array}{l}2007-2009 \\
\text { (construcción de } \\
\text { obras principales) }\end{array}$ & $\begin{array}{l}\text { Comunidades de } \\
\text { influencia, } \\
\text { campamentos y } \\
\text { frentes de obra }\end{array}$ & Trimestral & $\begin{array}{l}\text { Aedes aegypti } \\
\text { Mosquitos de } \\
\text { recipientes } \\
\text { domésticos } \\
\text { Lutzomyia } \\
\text { Anopheles }\end{array}$ & $\begin{array}{l}\text { Levantamiento de } \\
\text { índices } \\
\text { Muestreo larvario } \\
\text { con cucharón } \\
\text { Cuatro trampas } \\
\text { CDC* }^{*} \\
\text { Cebo humano } \\
\text { protegido dos } \\
\text { personas } \\
\text { Muestreo larvario } \\
\text { con cucharón } \\
\text { Cuatro trampas } \\
\text { CDC* } \\
\text { Cebo humano } \\
\text { protegido dos } \\
\text { personas }\end{array}$ \\
\hline
\end{tabular}

${ }^{*}$ : dos trampas CDC en comunidades de Anorí y Amalfi, y dos trampas CDC en frentes de obra 
y recolector. En cada tubo se colocaban máximo cinco larvas y la determinación taxonómica se hizo en el Laboratorio de Salud Pública de la Universidad de Antioquia.

Los índices de infestación se calcularon según Nelson, 1986 (11):

Índice infestación viviendas $=$ (número viviendas positivas/número viviendas inspeccionadas) X 100

Índice infestación depósitos $=$ (número depósitos positivos/número depósitos inspeccionados) x 100
Índice Breteau = (número depósitos positivos/total viviendas inspeccionadas) $\times 100$

\section{Resultados}

\section{Vigilancia de Aedes aegypti}

En los frentes de obra de los proyectos Porce II y Porce III, no se encontró infestación por Ae. aegypti; por lo tanto, los índices de infestación en todas las obras y campamentos de ambos proyectos fueron de $0 \%$, debido posiblemente a su ubicación eminentemente rural.

Cuadro 2. Especies identificadas de insectos de importancia en salud pública en el área de influencia del proyecto hidroeléctrico Porce II, Antioquia, Colombia, 1990

\begin{tabular}{|c|c|c|c|c|c|c|c|c|}
\hline \multirow{2}{*}{$\begin{array}{l}\text { Sitio de } \\
\text { muestreo }\end{array}$} & \multicolumn{2}{|c|}{ Lutzomyia } & \multicolumn{2}{|c|}{ Mosquitos silvestres } & \multirow[b]{2}{*}{ Adultos } & \multicolumn{3}{|c|}{ Anopheles } \\
\hline & Especie & Adultos & Especie & Larvas & & Especie & Larvas & Adultos \\
\hline Vereda La Carolina, & Lu. barretoi & 1 & Ae. angustivittatus & 1 & 1 & An. triannulatus & 27 & 20 \\
\hline 970 msnm & Lu. lichyi & 1 & Cx. coronator & 24 & & An. nuñeztovari & 19 & \\
\hline \multirow[t]{3}{*}{ (Gómez Plata) } & Lu. gomezi & 1 & $C x$. nigripalpus & 5 & & An. pseudopunctipennis & 10 & \\
\hline & & & $C x$. quinquefasciatus & 4 & & An. punctimacula & & 1 \\
\hline & & & Uranotaenia $s p$ & 3 & & & & \\
\hline Total & & 3 & & 37 & 1 & & 56 & 21 \\
\hline \multirow{4}{*}{$\begin{array}{l}\text { Vereda Los Puentes, } \\
1.000 \text { msnm } \\
\text { (Gómez Plata) }\end{array}$} & * & * & Ae. angustivittatus & & 6 & An. argyritarsis & & \\
\hline & & 1 & Ae. serratus & & 1 & An. nuñeztovari & 3 & \\
\hline & & & $\begin{array}{l}\text { Ae. fluviatilis } \\
\text { Culex sp. }\end{array}$ & 10 & & Cx. coronator & 15 & \\
\hline & & 2 & $\begin{array}{l}\text { Ma. juxtamansonia } \\
\text { Ps. ciliata }\end{array}$ & & $\begin{array}{l}6 \\
3\end{array}$ & & & \\
\hline Total & & & & 25 & 18 & & 4 & \\
\hline Corregimiento & * & * & Cx. coronator & 10 & & An. triannulatus & 6 & \\
\hline $\begin{array}{l}\text { La Cancana, } \\
1.000 \text { msnm } \\
\text { (Yolombó) }\end{array}$ & & & Cx. quinquefasciatus & 12 & & An. nuñeztovari & 5 & \\
\hline Total & & & & 22 & & & 11 & \\
\hline \multirow{11}{*}{$\begin{array}{l}\text { Sector El Bosque, } \\
1.000 \mathrm{msnm} \\
\text { (Amalfi) }\end{array}$} & Lu. lichyi & 23 & Hg. janthinomys & 10 & 17 & An. punctimacula & & 1 \\
\hline & Lu. gomezi & 1 & Sabethes sp. & & 1 & An. eiseni & & 1 \\
\hline & Lu. shannoni & 29 & Ae. angustivittatus & & 1 & & & \\
\hline & Lu. panamensis & 1 & Ae. scapularis & & 92 & & & \\
\hline & Lu. triramula & 114 & Aedes sp. & & 3 & & & \\
\hline & Lu. carrerai thula & 1 & Culex sp. & & 42 & & & \\
\hline & Lu. walkeri & 1 & Ma. juxtamansonia & & 1 & & & \\
\hline & & & Psorophora lutzii & & 9 & & & \\
\hline & & & Psorophora ferox & & 17 & & & \\
\hline & & & Wyeomyia sp. & & 2 & & & \\
\hline & & & Phoniomyia sp. & & 1 & & & \\
\hline Total & & 170 & & 10 & 186 & & & 2 \\
\hline Vereda Puente & Lu. lichyi & 33 & Ae. angustivittatus & & 3 & An. nuñeztovari & 21 & \\
\hline Porce, 800 & Lu. gomezi & 40 & Ae. scapularis & 2 & & An. pseudopunctipennis & 8 & 1 \\
\hline \multirow[t]{3}{*}{ msnm (Amalfi) } & Lu. shannoni & 1 & $C x$. coronator & 29 & & An. allopha & & 1 \\
\hline & Lu. triramula & 9 & Cx. quinquefasciatus & 10 & & An. neomaculipalpus & & 1 \\
\hline & Lu. carrerai thula & 4 & Culex sp. & & 30 & Wyeomyia sp. & & 6 \\
\hline Total & & 87 & & 39 & 41 & & 29 & 3 \\
\hline Vereda Guadalupe & Lu. gomezi & 8 & Aedes sp. & 1 & & An. pseudopunctipennis & 57 & \\
\hline IV Campamento & & & Cx. coronator & 13 & & & & \\
\hline Los Cedros, 750 & & & Cx. nigripalpus & 12 & & & & \\
\hline \multirow[t]{2}{*}{ msnm (Guadalupe) } & & & Culex sp. & & 5 & & & \\
\hline & & & Uranotaenia sp. & & 1 & & & \\
\hline Total & & 8 & & 25 & 7 & & 57 & \\
\hline
\end{tabular}

* No se colocó estación de muestreo en el sitio. 
En las comunidades del área de influencia de estos dos proyectos sólo se encontró infestación por $A e$. aegypti en el caserío La Cancana (Yolombó) y en caserío de la vereda La Bramadora (Guadalupe).

En el caserío La Cancana (Yolombó) del área de influencia de Porce II, ubicado a 1.000 msnm, se registraron índices de infestación de viviendas de $16,4 \%$ y de depósitos de 15,4\%. De estos últimos, la distribución porcentual de criaderos positivos fue: llantas, 31,8\% (7); tarros, 13,6\% (3); matas sembradas en agua, 13,6\% (3); canecas y bebederos, 9,1\% (2), cada uno, y otros $22,8 \%$ (5). Posteriormente, este caserío fue trasladado para ser inundado por el embalse, en 1998.

En el caserío de la vereda La Bramadora (Guadalupe) del área de influencia de Porce III, ubicada a $700 \mathrm{msnm}$, con índices de infestación de viviendas de 0,51\% y de depósitos de 0,29\% en el año 2003, donde, de 343 depósitos con agua (tanques y pozos de cianuro, llantas, bebederos y bañaderos para animales, canecas, tanques y galones, zanjas empozadas e inservibles), solo un sanitario en desuso se encontró positivo para este mosquito. Igualmente, entre los años 2005 y 2006, en los muestreos semestrales en este caserío, los índices de infestación de depósitos fueron inferiores a $4 \%$, donde, de 94 depósitos con agua, dos tanques, una caneca y una llanta estaban infestados con Ae. aegypti. Este caserío fue desalojado a finales de 2006 para ser trasladado a otra área del municipio. En las demás localidades estudiadas, los índices de infestación por $A e$. aegypti fueron $0 \%$.

\section{Vigilancia de otros mosquitos de recipientes}

Durante la construcción de Porce II, 1997-2000, dado que las comunidades más cercanas al área de influencia eran distantes de la construcción de la obra y a que fueron reubicadas en 1998, la vigilancia de vectores se centró en la búsqueda de criaderos en los campamentos y frentes de trabajo del proyecto hidroeléctrico, y en Porce III, en las veredas de influencia durante los levantamientos de índices de infestación para Ae. aegypti. Se encontraron principalmente larvas de mosquitos de géneros Culex, Aedes y Psorophora en recipientes domésticos abandonados y en pozos temporales de agua en las obras (cuadro 4). Ninguno de estos mosquitos, a excepción de Ae. aegypti hallado en la vereda La Bramadora, tiene importancia en la transmisión de arbovirosis en el departamento de Antioquia, pero son causantes de molestias por las picaduras frecuentes a las personas. El resultado positivo en larvas de mosquitos de recipientes, según el tipo de depósito, fue el siguiente: llantas $(31,9 \%)$, matas sembradas en agua y floreros $(16,7 \%)$, canecas $(7,5 \%)$, tanques bajos $(0,9 \%)$ y los denominados otros $(25,8 \%)$.

\section{Vigilancia de Lutzomyia spp.}

En el área de influencia de Porce II, en el levantamiento de especies que se llevó a cabo en 1990, antes de la construcción del proyecto, se identificaron ocho especies (cuadro 2), mientras que, en las recolecciones en Porce III, se identificaron 16 especies (cuadros 3 y 5 ).

\section{Vigilancia de anofelinos}

Esta fue exhaustiva en las comunidades ubicadas en el área de influencia de Porce II y en los campamentos, dado que es una zona aurífera y de baja pendiente, donde se encontraron especies de importancia en salud pública, como An. nuñeztovari y An. pseudopunctipennis (cuadro 2). Durante las capturas nocturnas en bosque, en Porce III, entre 2005 y 2006, se capturó un ejemplar de An. neivai y uno de $A n$. eiseni. No obstante, la recolección de larvas de An. pseudopunctipennis fue frecuente en la ribera plana del río Porce, en la vereda La Bramadora, lugar distante de la construcción de las obras principales. Entre 2007 y 2009, en la vereda El Retiro, se localizaron dos criaderos domiciliarios de $A n$. argyritarsis (en caneca y charca) y de $A n$. pseudopunctipennis.

\section{Vigilancia de otros mosquitos silvestres}

Durante el levantamiento de la fauna de insectos en 1990, antes de iniciarse la construcción de Porce II, se hallaron insectos de los géneros Aedes, Mansonia, Culex, Psorophora, Wyeomyia, Phonyomyia, Uranotaenia, Haemagogus y Sabethes. Además, entre los años 2004 y 2006, durante la construcción de obras civiles y de infraestructura de Porce III, se identificaron dos nuevos géneros en las capturas: Trichoprosopon y Limatus.

\section{Discusión}

Diferentes investigadores han reportado la aparición o el aumento de casos de malaria y de especies de anofelinos vectores, en áreas donde se han construido represas (12-15). Además, se ha reportado el aumento de otras especies de mosquitos vectores de arbovirosis, tales como Mansonia sp., Psorophora sp. y Culex sp., entre otros $(16,17)$. En este sentido, el levantamiento 
Cuadro 3. Especies de Lutzomyia según sitio de captura durante la construcción de las obras civiles y de infraestructura, proyecto hidroeléctrico Porce III, 2004-2006

\begin{tabular}{|c|c|c|c|c|c|c|c|c|c|c|}
\hline \multirow{2}{*}{$\begin{array}{l}\text { Municipio } \\
\text { Lugar }\end{array}$} & \multicolumn{3}{|c|}{ Amalfi } & \multicolumn{7}{|c|}{ Anorí } \\
\hline & 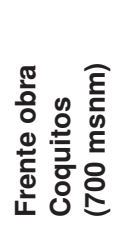 & 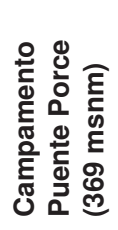 & 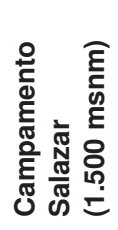 & 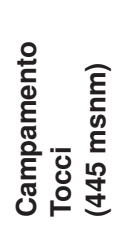 & 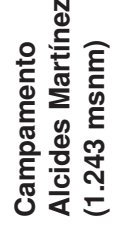 & 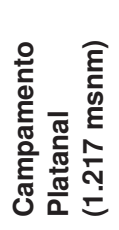 & 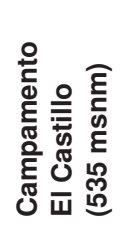 & 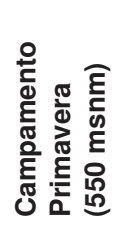 & 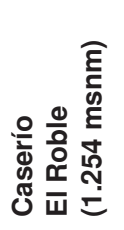 & $\begin{array}{l}\bar{\Phi} \\
\stackrel{ }{\circ}\end{array}$ \\
\hline L. carrerai & 4 & 9 & 2 & 69 & & & & & & 84 \\
\hline L. panamensis & & 20 & & 18 & & & & & & 38 \\
\hline L. yuilli & 1 & 36 & 8 & 39 & 160 & 16 & 5 & & 22 & 287 \\
\hline L. trapidoi & 1 & & & & & & & & 22 & 23 \\
\hline L. saulensis & & 1 & & 1 & & & & & 13 & 15 \\
\hline L. bifoliata & & & & 4 & 3 & & & & 2 & 9 \\
\hline L. hartmanni & & 15 & 3 & 15 & 6 & & & & 4 & 43 \\
\hline L. triramula & & 2 & & 27 & 1 & & & 1 & 1 & 32 \\
\hline L. gomezi & & 3 & & & & & & & & 3 \\
\hline L. caprina & & 1 & & & & & & & & 1 \\
\hline L. camposi & & & & 8 & & & & & & 8 \\
\hline L. aclydifera & & 1 & & 1 & & & & & 2 & 4 \\
\hline L. davisi & & & & 2 & & & & & & 2 \\
\hline Total & 6 & 88 & 13 & 184 & 170 & 16 & 5 & 1 & 66 & 549 \\
\hline
\end{tabular}

Cuadro 4. Especies de larvas de mosquitos de recipientes recolectadas en frentes de trabajo del proyecto hidroeléctrico Porce II y en veredas del área de influencia de Porce III

\begin{tabular}{|c|c|c|c|c|c|}
\hline \multirow[b]{2}{*}{ Especie } & \multirow{2}{*}{$\begin{array}{c}\text { Porce II } \\
1997-2000 \\
\text { Frentes de obra } \\
(820 \mathrm{msnm})\end{array}$} & \multicolumn{4}{|c|}{ Localidades del área de influencia de Porce III, 2005-2009 } \\
\hline & & $\begin{array}{l}\text { La Bramadora } \\
\text { (700 msnm) }\end{array}$ & $\begin{array}{c}\text { El Limón } \\
\text { (1.144 msnm) }\end{array}$ & $\begin{array}{c}\text { El Roble } \\
(1.237 \mathrm{msnm})\end{array}$ & $\begin{array}{c}\text { El Retiro } \\
(1.483 \mathrm{msnm})\end{array}$ \\
\hline Culex quinquefasciatus & & & $x$ & $x$ & $x$ \\
\hline Culex coronator & $x$ & $x$ & $x$ & $x$ & $x$ \\
\hline Culex nigripalpus & $x$ & $x$ & $\mathrm{X}$ & $x$ & $x$ \\
\hline Aedes aegypti & & $x$ & & & \\
\hline Culex corniger & $x$ & $X$ & $x$ & $x$ & $x$ \\
\hline Aedes fluviatilis & $x$ & & & & \\
\hline Psorophora ferox & $x$ & & & & \\
\hline Psorophora confinnis & $x$ & & & & \\
\hline Limatus durhami & $x$ & & $x$ & $x$ & $x$ \\
\hline Ortophodomyia sp. & & & & & $x$ \\
\hline Uranotaenia sp. & & $x$ & & & \\
\hline
\end{tabular}

de la fauna de especies y la vigilancia mediante muestreos periódicos y permanentes, antes y durante la construcción de plantas hidroeléctricas, son necesarios para prevenir o controlar el aumento de enfermedades de transmisión por vectores, como malaria, dengue, fiebre amarilla y otras arbovirosis, tal como se indica en este estudio.

La vigilancia de Ae. aegypti es importante, debido al desplazamiento que ha tenido hacia las zonas rurales del país (18), donde constituye una amenaza para expandir la frontera del dengue con el inicio de epidemias rurales, y causar incapacidad escolar y laboral o muerte por dengue grave. En el departamento de Antioquia, este vector predomina en las áreas urbanas hasta los 2.000 msnm (19). No obstante, los bajos índices de infestación de Ae. aegypti encontrados en la zona donde se ubican los proyectos hidroeléctricos Porce II y Porce III, hacen que el riesgo de transmisión de dengue en los trabajadores y en los habitantes de las comunidades sea mínimo, puesto que la infestación ha estado limitada, y la inundación del embalse Porce II y el reasentamiento de los pobladores en otras localidades contribuyeron a eliminar la infestación en la zona. Además, en las viviendas se tiene suministro de agua por 
acueductos de las veredas, lo que hace que las personas no almacenen agua en tanques y canecas. Sin embargo, la inadecuada disposición de los residuos sólidos en las veredas propicia espacios adecuados para la cría, situación que ha favorecido la presencia de recipientes domésticos que pueden albergar agua en temporada de lluvias $y$, por ende, favorecen la cría de mosquitos. Tal situación hace necesario mantener una vigilancia de los criaderos y las densidades de larvas y adultos en las viviendas del área de influencia y en frentes de obra, debido a que el aumento de las poblaciones de insectos podría llegar a causar problemas de salud pública.

Se destaca que el género de insectos de mayor importancia en la zona del complejo hidroeléctrico es Lutzomyia spp., transmisor de la leishmaniasis, enfermedad endémica en los municipios de Amalfi y Anorí donde se ubican los proyectos, lo que hace que la leishmaniasis constituya un riesgo permanente para los trabajadores. Entre 2008 y 2009, la incidencia de leishmaniasis en Anorí fue de 841 por 100.000 (83 casos) y 745,8 por 100.000 (74 casos) habitantes, y en Amalfi, de 249 por 100.000 (24 casos) y 196,7 por 100.000 (19 casos) habitantes, respectivamente (20).

No obstante, a pesar del carácter endémico de la enfermedad en los municipios, la leishmaniasis no fue causa de morbilidad en los trabajadores de los frentes de obra de Porce II ni en el área de influencia, debido a que los campamentos y frentes de trabajo no se encontraban ubicados en lugares cercanos a bosques. Esta situación fue contraria en Porce III, donde se presentaron los casos, puesto que el lugar posee bosques en la zona de las obras y peridomiciliarios.

Los sitios de muestreo en Porce III tuvieron algunas variaciones entre las obras de infraestructura y las obras principales, debido a la ubicación de los campamentos de trabajadores (zonas más despejadas de cobertura vegetal durante la construcción de obras principales) y a la concentración de los trabajos en la presa y la casa de máquinas.

El hallazgo de potenciales especies vectoras, como Lutzomia trapidoi, Lu. hartmanni y Lu. panamensis, podría explicar la presencia de nueve casos de leishmaniasis entre los trabajadores de Porce III en el año 2005, para una incidencia de 3,9 por 1.000 trabajadores y seis casos en los habitantes de la vereda El Roble, aledaña al proyecto hidroeléctrico. En el año 2008, se diagnosticaron cinco casos entre los trabajadores y dos casos en la misma vereda. De igual forma, en el año 2009, se reportaron 14 casos en trabajadores, de los cuales 11 fueron considerados autóctonos del área donde se estaba

Cuadro 5. Especies de Lutzomyia según sitio durante la construcción de las obras principales (presa y casa de máquinas) del proyecto hidroeléctrico Porce III, 2007-2009

\begin{tabular}{|c|c|c|c|c|c|c|c|}
\hline \multirow[t]{2}{*}{ Municipio } & \multicolumn{2}{|c|}{ Amalfi } & \multicolumn{4}{|c|}{ Anorí } & \multirow[b]{2}{*}{ స్ } \\
\hline & 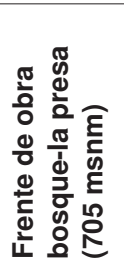 & 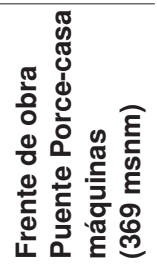 & 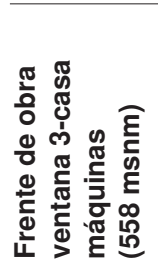 & 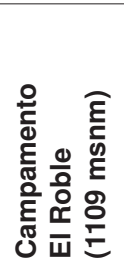 & 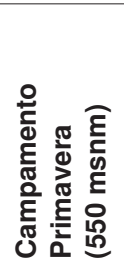 & 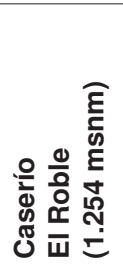 & \\
\hline L. carrerai & 12 & 1 & 130 & 8 & & 7 & 158 \\
\hline L. scorzai & 14 & 7 & 57 & 3 & & 1 & 82 \\
\hline L. panamensis & & & 30 & & & 2 & 32 \\
\hline L. yuilli & 1 & 7 & 20 & & 2 & & 30 \\
\hline L. trapidoi & 3 & & 7 & & & & 10 \\
\hline L. saulensis & & 7 & & & & & 7 \\
\hline L. cellulana & & 1 & 5 & & & & 6 \\
\hline L. bifoliata & 2 & & & & 2 & & 4 \\
\hline L. hartmanni & 4 & & & & & & 4 \\
\hline L. triramula & & & 4 & & & & 4 \\
\hline L. micropyga & 2 & & & & & & 2 \\
\hline Lutzomyia sp. & 4 & & 15 & & & 2 & 21 \\
\hline Total & 42 & 23 & 268 & 11 & 4 & 12 & 360 \\
\hline
\end{tabular}


construyendo Porce III, obteniéndose una incidencia de 3,8 por 1.000 trabajadores. En las veredas de la zona se reportaron adicionalmente ocho casos. La información sobre casos de leishmaniasis en trabajadores fue tomada del Sistema de Vigilancia Epidemiológica específico de Porce III.

Específicamente para Colombia, Lu. trapidoi y Lu. hartmanni se consideran vectores primarios de leishmaniasis (21,22); ambas especies podrían estar incriminadas en la transmisión de leishmaniasis en la zona. Lutzomyia trapidoi fue encontrada en el bosque cercano a la presa en margen derecha, en el frente de obra Ventana 3 y en el caserío Pueblo Nuevo de la vereda El Roble; Lu. hartmanni fue hallada en el bosque de presa margen derecha, y en el campamento Salazar y Puente Porce.

En todas las capturas realizadas en el bosque de Ventana 3 y el campamento Tocci, se obtuvo el mayor número de ejemplares y la mayor diversidad de especies, situación que se podría explicar, en parte, debido a que estos sitios conservan abundante cobertura vegetal, propiciando abrigo, humedad y temperatura adecuada a los insectos y corredores biológicos de dispersión entre los sitios de cría del flebotomino, los mamíferos reservorios y la población humana; estos aspectos, a su vez, influyen en la eco-epidemiología de la transmisión de la leishmaniasis (Ferro C, Fuya P, Pérez S, Lugo L, González C. Valoración de la ecoepidemiología de las leishmaniasis en Colombia a partir de la distribución espacial y ecológica de los insectos vectores. Biomédica. 2011;31 (Supl.3):315). Las especies más abundantes en los muestreos fueron Lu. carrerai, Lu. yuilli y Lu. scorzai. En algunos reportes, Lu. yuilli se ha encontrado infectado con Leishmania spp. y se discute su importancia en la transmisión de la enfermedad (23); esta especie fue encontrada en el bosque de casa de máquinas en Ventana 3, en el bosque de la presa en margen derecha, en Puente Porce y el campamento La Primavera.

Es importante tener en cuenta que en el inventario de fauna silvestre elaborado por el personal de la Corporación Ambiental de la Universidad de Antioquia, se documentó la presencia de mamíferos silvestres de las especies Didelphis marsupialis (zarigüeya), Tamandua mexicana y Cyclopes didactylus (osos hormigueros), Bradypus variegatus (perezoso), Dasypus novemcintus ("gurre" o armadillo), Cerdocyon thous (zorro), Potos flavus (perro de monte) y roedores pertene- cientes a seis familias y ocho especies, los cuales se han descrito como reservorios de leishmaniasis en el país y en América Latina, lo que indica el riesgo inminente en la zona para la enfermedad (24-26).

De las especies de Anopheles identificadas en la zona, las de mayor importancia en salud pública son An. nuñeztovariy An. pseudopunctipennis, descritos como vectores de malaria para Colombia (27).

Durante la construcción del proyecto hidroeléctrico Porce II, solo se presentó un brote epidémico de malaria que afectó a seis trabajadores y fue controlado con el apoyo de la Secretaría de Salud del municipio de Yolombó, registrado por los mismos investigadores (28).

No obstante, la malaria no representó un problema de importancia en salud pública en el área de influencia de Porce III, debido a que en la zona predominan los terrenos de alta pendiente, lo cual impide que el agua se empoce. De tal manera, las capturas de ejemplares adultos de anofelinos fueron esporádicas durante los seis años de vigilancia entomológica, estando casi totalmente ausentes en los muestreos nocturnos con trampas de luz y cebo humano protegido, en los bosques, campamentos y frentes de obra.

El hallazgo frecuente de An. pseudopunctipennis en la vereda La Bramadora, con ocupación minera y en su mayoría de explotación de veta aurífera cerca al río Porce donde se encuentran algunas lagunas de inundación, podría explicar, en parte, la presencia de los dos casos de malaria durante la construcción del proyecto Porce III, en el 2006.

Con relación a las especies de otros mosquitos silvestres capturados en Porce II y Porce III, los de mayor importancia en salud pública en Colombia son los géneros Haemagogus y Sabethes, teniendo en cuenta que algunas especies pueden ser transmisores eficientes de fiebre amarilla selvática. La principal especie capturada en bosques aledaños a ambos proyectos fue Haemagogus janthinomys, considerado el principal vector de fiebre amarilla en Colombia y en América Latina (29). Este hallazgo conllevó a Empresas Públicas de Medellín a exigir la vacunación contra esta enfermedad en los trabajadores, antes de su ingreso a laborar en el proyecto.

Los mosquitos de los géneros Psorophora, Mansonia y Culex han sido registrados como de importancia en la transmisión de arbovirosis en Colombia, principalmente de la encefalitis equina 
venezolana (30, Olano V. La encefalitis venezolana en La Guajira, Colombia, 1995. Biomédica. 1995; 15(Supl.1).

Los hallazgos anteriores permitieron hacer las recomendaciones pertinentes a las Empresas Públicas de Medellín y a las empresas constructoras de las obras, para la prevención y el control de las enfermedades transmitidas por vectores en todos los campamentos y frentes de obra, tal como ha sido sugerido por algunos autores (31). Las principales acciones que se implementaron, fueron:

1) vigilancia epidemiológica activa para la detección oportuna de casos sospechosos de leishmaniasis 0 de otras enfermedades transmitidas por vectores;

2) disminución del contacto vector-hombre mediante el uso de repelentes, cubrimiento de espacios con anjeos en puertas y ventanas, y control de basureros para tener alejados a los reservorios silvestres de leishmaniasis;

3) control de pozos naturales de agua en los lugares cercanos a las obras de infraestructura para limitar los sitios de reproducción de zancudos;

4) vigilancia interna permanente sobre los depósitos artificiales para evitar la infestación de mosquitos;

5) vacunación para fiebre amarilla en todas las personas que laboraban en los proyectos Porce, $\mathrm{y}$

6) aplicación de insecticidas de tipo de volumen ultrabajo (ultra low volume, ULV) ante la aparición de varios casos de leishmaniasis en trabajadores del mismo frente de obra, donde se identificaron especies vectores y se presumía que se estaba activando un foco de la enfermedad.

Se concluye que, durante la construcción del proyecto Porce II y hasta el momento, el mayor riesgo de transmisión se presenta para malaria debido a la presencia constante de An. nuñeztovari y An. pseudopunctipennis, más aún, cuando ya se encuentra presente el cuerpo de agua del embalse de la central hidroeléctrica, inundada desde 1998. En la zona de la construcción del proyecto Porce III, el mayor riesgo de transmisión se presenta para leishmaniasis, debido a la presencia de especies de reconocida capacidad vectorial en Antioquia, como Lu. trapidoi y Lu. hartmanni, y a la circulación del parásito entre los animales silvestres que hacen presencia en el área, así como a las características de las labores que se realizan en el proyecto en horario nocturno.

Se destaca la importancia de la vigilancia periódica y permanente en las zonas donde se construyan proyectos hidroeléctricos en Colombia, dado que las características propias de la zona tórrida tropical permite la presencia de amplia diversidad de especies de importancia en salud pública, que podrían ocasionar riesgos para la salud y transmisión de enfermedades, no sólo a los trabajadores de las obras sino también a las comunidades aledañas.

\section{Agradecimientos}

A las Empresas Públicas de Medellín, a los habitantes de las comunidades y a los trabajadores de los proyectos Porce II y Porce III.

\section{Conflicto de intereses}

Los autores del artículo hacen constar que no existe, de manera directa o indirecta, ningún tipo de conflicto de intereses que pueda poner en peligro la validez de lo comunicado.

\section{Financiación}

El proyecto fue financiado por las Empresas Públicas de Medellín, mediante contratación con la Universidad de Antioquia para el desarrollo del Sistema de Vigilancia Epidemiológica en el área de influencia de los proyectos hidroeléctricos del río Porce.

\section{Referencias}

1. Schoijet M. Una introducción a la problemática de los impactos de las represas. En: Schorr T, editor. Las represas y sus efectos sobre la salud. México D.F.: Organización Panamericana de la Salud; 1984. p. 72-87.

2. Valencia ML, Escobar JP, López YL, González MC, Salazar B, Henao N, et al. Vigilancia de la morbilidad en los municipios del área de influencia durante la construcción del proyecto hidroeléctrico Porce II (Empresas Públicas de Medellín, ESP), Antioquia, Colombia, 1997-2000. Revista de la Facultad Nacional de Salud Pública. 2003;21:49-61.

3. Escobar JP, López Y, Wolff M, Gallo O, Osorio L. Insectos de importancia médica en la zona de influencia del proyecto hidroeléctrico Porce II. Medellín: Universidad de Antioquia, Facultad Nacional de Salud Pública; 1990. p. 75.

4. Cortés LA, Fernández JJ. Especies de Lutzomyia en un foco urbano de leishmaniasis visceral y cutáneo en el Carmen de Bolívar, Bolívar, Colombia. Biomédica. 2008;28:433-40

5. González R, Carrejo NS. Introducción al estudio taxonómico de Anopheles de Colombia. Segunda edición. Cali: Universidad del Valle; 2009. p. 178 
6. Lane J. Neotropical Culicidae. Sao Paulo: Universidad de Sao Paulo; 1953. p. 1112.

7. Tinker M. Clave práctica para las larvas de mosquitos neotropicales en recipientes. Bogotá: OPS/OMS; 1980.

8. Young D. A review of the bloodsucking Psychodid flies of Colombia. Univ Florida Agric Exp Stn Tech Bull. 1979;806:1266.

9. Forattini OP. Culicidologia Médica. Volume 2. Sao Paulo: Universidad São Paulo; 2002. p. 859.

10. Walter Reed Byosystematics [WRBU]. Unit 2001. Systematic catalog of Culicidae. Fecha de consulta: 19 de noviembre de 2011. Disponible en: http://www. mosquitocatalog.org

11. Nelson M. Aedes aegypti: biología y ecología. Washington: OPS/PNSP86-63; 1986. p. 50.

12. van Thiel PH. Malaria problems arising from the construction of reservoir in the interior of Surinam. Trop Geogr Med. 1962;14:259-78.

13. Worthington EB. Some ecological problems concerning engineering and tropical diseases. Engineering and Tropical Diseases. 1979;11:5-11.

14. Osorio Quintero L, Dutary Thatcher B, Tadei WP. Biología de anofelinos amazônicos. XXI. Ocorrência de espécies de Anopheles e outros culicídeos na hidrelétrica de Balbina cinco anos após o enchimento do reservatório. Acta Amazônica. 1996;26:281-96.

15. Paula M, Gomes A. Culicidae (Diptera) em área sob influência de construção de represa no estado de São Paulo, Brasil. Rev Saúde Pública. 2007;41:284-9. http:// dx.doi.org/10.1590/S0034-89102006005000018

16. Junk WJ, Nunes de Melo JA. Impactos ecológicos das hidroeletricas na bacia amazónica brasilera. En: Kolhepp G, Schrader A, editor. Homem e Natureza na Amazonia. Tübingen: Geographisches Institut; 1987. p. 367-85.

17. Tadei WP, Santos JM, Scarpassa VM, Rodríguez IB. Incidencia, distribuição e aspectos ecológicos de espécies de Anopheles (Diptera: Culicidae), em regiões naturais e sob impacto ambiental da Amazônia brasileira. In: Ferreira G, Jorge $\mathrm{E}$. Bases científicas para estrategias de preservaçao e dessenvolvimento da Amazonia. Mannaus: Inpa; 1993. p. 167-96.

18. Morales A. Aedes aegypti en zona rural del municipio de La Mesa, (Cundinamarca) Colombia. Biomédica. 1981;1:223-4.

19. López Y, Wolff M, Escobar JP, Osorio Q. Distribución espacial y hábitats larvarios de Aedes aegypti en el departamento de Antioquia. Revista Colombiana de Entomología. 1990;18:63-72.

20. Dirección Seccional de Salud de Antioquia. Eventos de salud pública por municipio en Antioquia, 2007-2010. Fecha de consulta: 19 de noviembre de 2011. Disponible en:http//:www.dssa.gov.co/index.php/estadisticas/eventosde-salud-publica
21. Morales A, Corredor A, Cáceres E, lbagos A, Rodríguez C. Aislamiento de tres cepas de Leishmania a partir de Lutzomyia trapidoi en Colombia. Biomédica. 1981;1:198207.

22. Duque P, Vélez I, Morales M, Sierra D. Sand flies fauna involved in the transmission of cutaneous leishmaniasis in Afro-Colombian and American communities of Chocó, Pacific coast of Colombia. Neotrop Entomol. 2004;33:25564. http://dx.doi.org/10.1590/S1519-566X2004000200018

23. Santamaría E, Ponce N, Zipa Y, Ferro, C. Presencia en el peridomicilio de vectores infectados con Leishmania (Viannia) panamensis en dos focos endémicos en el occidente de Boyacá, piedemonte del valle del Magdalena medio, Colombia. Biomédica. 2006;26(Supl.1):82-94

24. Laison R, Shaw JJ, Silveira FT, De Souza AA, Braga RR, Ishikawa EA. The dermal leishmaniosis, with special emphasis to de eco-epidemiology of the disease in Amazonia. Mem Inst Oswaldo Cruz. 1995;89:435-43. http:// dx.doi.org/10.1590/S0074-02761994000300027

25. Travi BL, Arteaga LT, Leon AP, Adler GH. Susceptibility of spiny rats (Proechimys semispinosus) to Leishmania (Viannia) panamensis and Leishmania (Leishmania) chagasi. Mem Inst Oswaldo Cruz. 2002;97:887-92. http:// dx.doi.org/10.1590/S0074-02762002000600025

26. Brandão Filho S, Brito ME, Francisco GC, Edna Al, Cupolillo E, Floeter Winter L, et al. Wild and synanthropic hosts of Leishmania (Viannia) braziliensis in the endemic cutaneous leishmaniasis locality of Amaraji, Pernambuco State, Brazil, 2003. Trans R Soc Trop Med Hyg. 2003;97:291-6.

27. Olano VA, Brochero HL, Sáenz R, Quiñones ML, Molina J. Mapas preliminares de la distribución de especies de Anopheles vectores de malaria en Colombia. Biomédica. $2001 ; 21: 402-8$.

28. González C, Ríos C, López Y, Osorio L, Valencia M, Escobar JP. Vigilancia de riesgos ambientales para la salud en la zona de construcción del proyecto hidroeléctrico Porce II. Antioquia, Colombia. 1997-2000. Revista Gestión y Ambiente. 2002;5:111-21.

29. Vidales H, Buitrago B, Sanín LH, Morales A, Groot H. Estudio de un brote epidémico de fiebre amarilla selvática en el piedemonte de la Sierra Nevada de Santa Marta, 1979. Biomédica. 1981;1:171-86.

30. Sanmartín C, Mackenzie RB, Trapido $\mathbf{H}$, Barreta $\mathbf{P}$, Mullenax $\mathrm{CH}$, Gutiérrez $\mathrm{S}$, et al. Encefalitis equina venezolana en Colombia, 1967. Bol Of Sanit Panam. 1973;74:108-37.

31. Birley M. Directrices para prever las consecuencias de las obras de desarrollo de los recursos hídricos en cuanto a las enfermedades transmitidas por vectores. Ginebra: Organización Mundial de la Salud; 1992. p. 20. 